\title{
The Need For a Universal Language and Methods of Its Creation as Suggested by Hangul
}

\author{
Myung-Gun Choo
}

Sejong institution

In 1994, the entire world watched in awe as a comet slammed into Jupiter and the event caused a major paradigm shift in the way mankind viewed the history of life on earth. We had come to realize that the ecosystem had not evolved along a smooth and continuous path to become its current form but rather that it had undergone a series of dramatic changes following several periodic extinctions. For many years a number of scientists had claimed that the collision of a comet and the earth drove the dinosaurs into extinction some 65 million years ago. While this view was regarded as just another hypothesis until recently, the event in 1994 shattered all opposing views and it is now accepted as an established theory. This implies that while life on earth began 4.6 billion years ago from a simple cell division, through both continuous evolution and periodic extinctions a great diversity of life forms at various stages of evolutionary processes have come to coexist in the world today.

Hence, people all over the world share a common origin and are close and precious brothers in this vast ocean of biological diversity. As recently as four million years ago, our ancestors lived together in Equatorial Africa. The end of the Ice Age made travel possible and in groups they scattered across the Temperate Zone. Settling into different areas each group developed its unique culture and form of language. However, even today we can discover similarities among different languages which is testimony to the fact that people across the world are all brothers sharing one common root. For example, the descendants of the Saxons who moved to England from Saxony in German still use house and father, two commonly used German words. Also, similarities can be found in the languages used by the Koreans and the Japanese who had originally moved to Japan from the Korean peninsula. 
Throughout human history the development of unique and separate cultures in physically distant regions had deepened language gaps. Yet, with the amazing advances made in the field of science and technology the world is rapidly becoming one huge village and physical distances are no longer obstacles to global interaction. As recently as two hundred years ago, the only mode of travel was by horses and horse-drawn carriages. A mere two centuries later, jet planes move people across the globe at 1,000 km per hour which implies that conceptually the world has shrunk to one hundredth of its former size in terms of distance. Furthermore, no matter how powerful and wealthy a nation might be, in today's world it cannot survive without trading with other countries. This eventually led to the birth of WTO which broke down barriers between countries and the world has become one in the economic sense as well.

In the area of international currency, the IMF wields more power than any single nation, including the United States, and ultranational organizations such as the World Bank and ICAO play major roles in bringing the world into one global community, sharing a common future. Also, in the areas of information and communication, the development of the Internet is bringing the world together at an explosive speed as even people in remote corners of the globe have the means to give and receive a huge amount of information cheaply and instantly.

Following the massacre of 70 million precious lives in two global tragedies that was World War I and II, an ultranational United Nations was born and the organization is a driving force in bringing the world together politically. In sports, the countries of the world truly become one community every four years as athletes from all corners of the globe become fellow participants in the Olympic Games. Also, music and arts, like sports, transcend language barriers and in these areas walls between countries are being torn down. However, languages have evolved over a long period of time to become their current forms and therefore, the incompatibility among different languages is the most difficult obstacle the world must overcome to build a truly global community.

We are forced to spend an exorbitant amount of time and money to learn another language just to be able to communicate with our foreign counterparts. Furthermore, minor misunderstandings arise all the time due to inefficient communication and sometimes these can lead to serious disputes among the parties involved. Also, the degrees of 
economic, scientific and technological progress have more or less been leveled and more countries, and consequently their languages, are on an equal footing than any other time in history. Hence, the number of different combinations needed in language-to-language translations has grown astronomically. While computers may be able to perform automatic translations it is on an extremely limited scale and for efficient translations among the many languages of the world to take place, a new form of language that can act as an intervening medium is needed.

Americans and those of Korean descent who reside in countries where English is the mother tongue often believe that English is an ideal candidate for a universal language. Their claim is based on the belief that the United States is one of the most advanced countries in the fields of science and technology and enjoys a commanding political, military and economic superiority. However, even a short trip to other countries or a careful observation of the political and economic changes around the world should easily prove how unrealistic this view is. It is becoming increasingly more difficult to use English in countries like France and Germany. Also, while the United States was once accountable for more than $50 \%$ of the global economy, that percentage has been on a continuous decline and presently stands at a mere $20 \%$. If the current trend continues, by the beginning of the 21st century the Asian countries will account for $40 \%$ of the global economy while the relative importance of the US continues to shrink. Also, it is close to impossible for traditionally proud countries such as China and Japan to accept English as a universal language just as it is for US to accept Chinese as one. This clearly illustrates the fact that any existing language cannot be accepted as a candidate for universal language since it automatically delineates first and second class citizens along linguistic lines.

It was in light of these facts that many pioneers including Jamenhof created universal languages such as the Esperanto and tried to popularize its use. Unfortunately, their efforts were not successful. Their objectives and philosophies were sound and I strongly believe that the reason for the failures were due to the imperfect method of implementation. For example, the grammar of Esperanto is complicated like Latin and relies heavily on inflections. These and the fact that the limited vocabulary consists mostly of words from Eastern European countries were major obstacles to it being accepted as a universal 
language.

Therefore, the creation of a true universal language requires the participation of all the different linguistic groups of the globe and the worldwide use of the language cannot be realized without their assistance. In this aspect, we can turn to history and learn from the example of the creation of Hangul. The Korean alphabet Hangul was first created in 1446. Its widespread dissemination was disrupted due to the chaotic domestic situation following the usurpation of the crown by King Sejo. The alphabet survived barely among the peasant classes and it was not until 1910 after the fall of the Chosun Dynasty that Hangul was revived and widely used. Over a short period of 40 years, the use of the alphabet spread rapidly across the peninsula and with the liberation of Korea from Japanese occupation. Hangul was firmly established as the national alphabet of the country.

This one example teaches us that any language and alphabet capable of satisfying the needs of the populace can be revived even after being virtually unused for 500 long years. Also, while the creation of Hangul was not necessarily the birth of a new language, its method of marking sounds was easily learnable even by the simplest people. The illiterate poor who had been deprived of opportunities to communicate due to lack of time and money wholeheartedly embraced the creation of Hangul and their support and continued use were the reasons that it was able to survive and indeed flourish.

Therefore, the first step in the creation of a universal language is to select words, starting with those that are most frequently used in our daily lives. Eventually comprehensive list should include all the important words and it should be emphasized that they are the common property of all the people of the world. Well-directed balancing of languages with an abundant stock of words and those more limited, will enrich the vocabulary and people the world over will be able to enjoy a broader and richer level of thought through the use of a universal language. Each culture had evolved separately in its own unique surroundings and therefore, in some areas it exhibits a higher level of sophistication compared to other cultures. For example, the Eskimos exhibits a higher level of sophistication compared to other cultures. For example, the Eskimos who are among the least advanced ethnic groups in virtually every aspect, have the most expansive selection of words used to categorize the different types of snow. Koreans have a virtually limitless repertoire of echoic and mimetic words by utilizing vowel 
gradations. Also, in the areas of computers and aeronautical engineering, English is the ideal choice while in philosophy, medical science and compound words. Latin and Greek are absolutely the more superior languages. Similarly, French is the ideal language for fine arts and in music, German and Italian are the appropriate choices. Therefore, by systematically and logically combining the strengths of these various languages, we can raise the level of human thought to new heights and embark on a whole new chapter in history of human culture.

With the marvelous advances in transportation technology and the rapid rise in disposable income, the annual figure for international travelers is well over 500 million and is expected to reach one billion by the beginning of the 21st century. These are the people most affected by the lack of a universal language and who must confront on a daily basis the inconveniences and discriminations brought on by their inability to communicate in a foreign language. One of man's strongest instincts is to explore his surroundings and to continuously strive to expand his horizons, both literally and conceptually. Without this, he would merely be surviving and not truly living.

Therefore, it is safe to assume that the number of people traveling to expand their horizons continues to increase dramatically and the time will come when the freedom to travel will be firmly established as one of the basic rights of a citizen of the global village.

The two main obstacles in international travel are the visa requirements and language barriers. Fortunately the first obstacle is becoming less of a hindrance as several advanced countries grant novisa privileges, albeit on a limited and highly selective basis. However, the language problem is becoming even more serious as nationalist sentiments continue to grow stronger. The international airports of today resemble exhibit halls with pictograms are no longer available. Therefore, an international language will provide them with the means to make a unified world a reality. And this, I strongly believe will eventually lead to a unified global nation with one language and one legislation. 